\title{
Depression as the First Manifestation in a Young Girl With Juvenile Systemic Lupus Erythematosus
}

\author{
Tania Caroline MONTEIRO DE CASTRO, ${ }^{1}$ Ho Chi HSIEN ${ }^{2}$ \\ ${ }^{1}$ Department of Pediatrics, Hospital São Camilo, São Paulo, Brazil \\ ${ }^{2}$ Department of Nephrology, Hospital São Camilo, São Paulo, Brazil
}

Juvenile systemic lupus erythematosus (JSLE) is a multisystem autoimmune disease in which autoantibodies target many body organs, including the central nervous system. Neuropsychiatric manifestations can occur in $22-95 \%$ of pediatric cases, but are much less frequent as an initial clinical event, rarely leading to a diagnosis of JSLE. $^{1-3}$ Depression can be a symptom of active JSLE or it can occur as a reaction following diagnosis of chronic illness. ${ }^{4-7}$

A 12-year-old girl was admitted with a history of sadness, decreased interest in activities, poor communication, and absences from school, fatigue, irritable moods, and arthralgia in her hands. The patient had not yet had menarche. The symptoms worsened, so the family decided to call for a psychiatric consultation. Given a diagnosis of depression, she was started on treatment with antidepressants without any response. Her depressive symptoms continued, but the family decided not to seek continued medical help. As stated above, after waiting for over a year, she was admitted to another hospital with nephrotic syndrome, anasarca, and a positive antinuclear antibody.

Prednisone $1 \mathrm{mg} / \mathrm{kg} /$ day was started and after 30 days, little improvement was seen. She was then transferred to our hospital with periorbital edema and pleural effusion. Urinary analysis revealed microscopic hematuria and proteinuria
(24 hour proteinuria was $3814 \mathrm{mg} / \mathrm{L}$ ) with normal renal function. A written informed consent was obtained from the parents or guardians.

Other laboratory findings included hemoglobin, $7.6 \mathrm{~g} / \mathrm{dL}$; white cell count, $10,360 / \mathrm{mm}^{3}$ (polymorphonuclear leukocyte count of $9,220 / \mathrm{mm}^{3}$; lymphocyte count of $\left.932 / \mathrm{mm}^{3}\right)$; and platelet count, $356,000 / \mathrm{mm}^{3}$. Erythrocyte sedimentation rate of $120 \mathrm{~mm}$ in the first hour with C-reactive protein was $19.0 \mathrm{mg} / \mathrm{dL}$. Immunological investigation revealed antinuclear antibodies 1:640 homogeneous pattern, antidouble stranded deoxyribonucleic acid antibodies $316 \mathrm{UI} / \mathrm{mL}(<100 \mathrm{UI} / \mathrm{mL})$, and low serum C3 and C4 complement levels.

Renal biopsy showed diffuse proliferative glomerulonephritis, class IV-S (A/C) International Society of Nephrology/Renal Pathology Society, 2004, with activity index of 6 and chronicity index of 2 (Figures 1 and 2). Immunofluorescence staining showed deposits of immunoglobulin $\mathrm{M}$, immunoglobulin $\mathrm{G}$, immunoglobulin $\mathrm{A}$, as well as $\mathrm{C} 3$ and $\mathrm{C} 1 \mathrm{q}$ in mesangium and capillary loops, with segmental and diffuse distribution. Lupus nephritis was considered, as the patient fulfilled the diagnostic criteria for SLE.

The dose of prednisone was increased to $2 \mathrm{mg} / \mathrm{kg} /$ day and pulse with methylprednisolone was started. There was markedly improved 

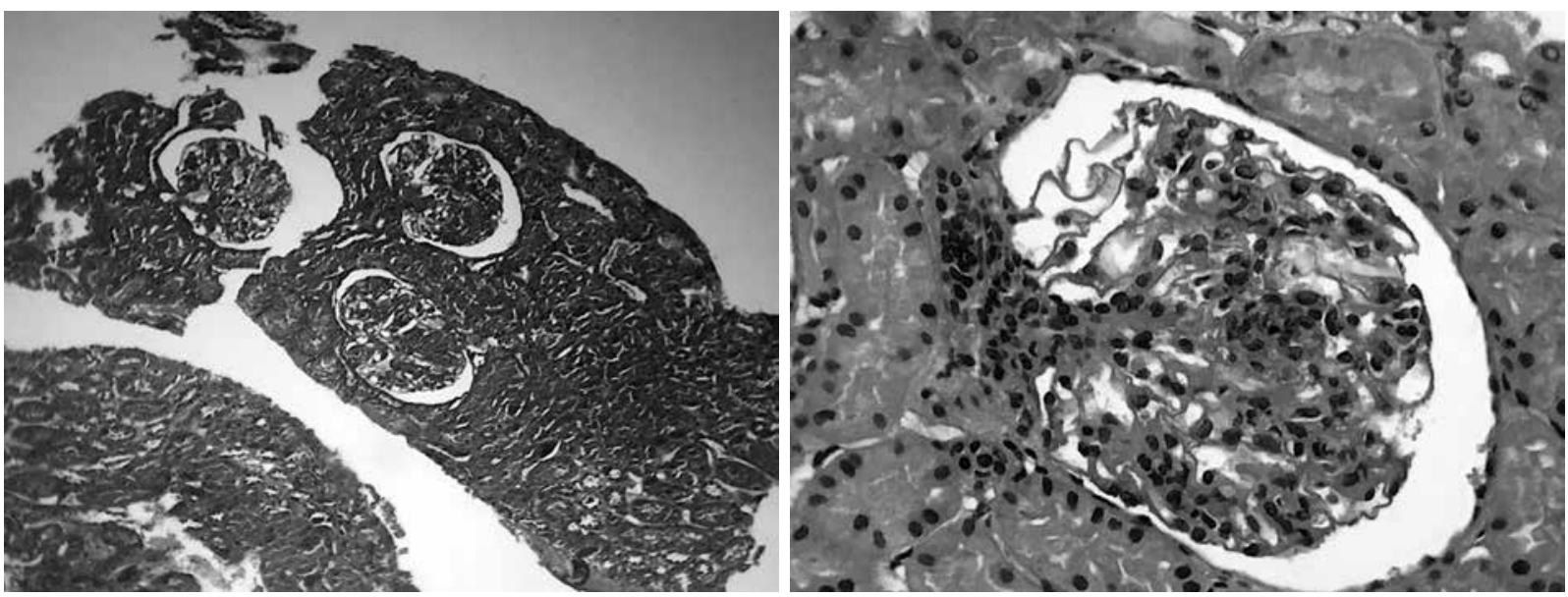

Figure 1. Tufts reveal segmental proliferation of mesangial cells and endocapillar with segmental expansion of mesangial matrix and formation of synechiae. There is focal karyorrhexis. Basement membrane does not present significant alterations. Capillary loops have a "wire loop"component.

clinical response with regression of the depression and anasarca. Cyclophosphamide and methylprednisolone were added, while maintaining continuous oral prednisone.

She had no recurrence of depression during the four-month period follow-up. This case report illustrates the diagnostic challenge in juvenile neuropsychiatric lupus. Early diagnosis and precocious treatment may be important to control disease sequelae and reduce the high morbidity of this disease.

Our case highlights the need for rheumatologists to recognize that depression, in a young female patient without response to antidepressants, could be the first manifestation of JSLE. Identification of SLE-specific biomarkers of depression also has high priority.

\section{Declaration of conflicting interests}

The authors declared no conflicts of interest with respect to the authorship and/or publication of this article.

\section{Funding}

The authors received no financial support for the research and/or authorship of this article.

\section{REFERENCES}

1. ACR Ad Hoc Committee on Neuropsychiatric Lupus Nomenclature.The American College of Rheumatology nomenclature and case definitions for neuropsychiatric lupus syndromes . Arthritis Rheum 1999;42:599-608.

2. Ferraria N, Rocha S, Fernandes VS, Correia T, Gonçalves E. Juvenile systemic lupus erythematosus with primary neuropsychiatric presentation. BMJ Case Rep 2013;2013. pii: bcr2012008270

3. Kohut SA, Williams TS, Jayanthikumar J, LandoltMarticorena C, Lefebure A, Silverman E, et al. Depressive symptoms are prevalent in childhoodonset systemic lupus erythematosus (cSLE). Lupus 2013;22:712-20.

4. Palagini L, Mosca M, Tani C, Gemignani A, Mauri M, Bombardieri S. Depression and systemic lupus erythematosus: a systematic review. Lupus 2013;22:409-16.

5. Demirkaya E, Bilginer Y, Aktay-Ayaz N, Yalnizoğlu D, Karli-Oğuz K, Isikhan V, et al. Neuropsychiatric involvement in juvenile systemic lupus erythematosus. Turk J Pediatr 2008;50:126-31.

6. $\mathrm{Yu} \mathrm{HH}$, Lee $\mathrm{JH}$, Wang LC, Yang $\mathrm{YH}$, Chiang BL. Neuropsychiatric manifestations in pediatric systemic lupus erythematosus: a 20-year study. Lupus 2006;15:651-7.

7. Hadeel MAR, Haitham MH, Rehab AK. Psychiatric disorders in juvenile systemic lupus erythematosus: prevalence and association with autoantibodies. Middle East Curr Psychiatry 2012;19:48-55. 